\title{
Amélioration de la valeur nutritionnelle des gousses de Piliostigma reticulatum (D. C.) Hochst dans l'alimentation du bétail en période de soudure
}

\author{
Sita SANOU ${ }^{1 *}$, Louis SAWADOGO ${ }^{2}$ et Chantal-Yvette KABORE-ZOUNGRANA ${ }^{3}$ \\ ${ }^{1}$ Laboratoire d'Etudes et de Recherches des Ressources Naturelles et des Sciences de l'Environnement \\ (LERNSE), Université Polytechnique de Bobo-Dioulasso (UPB), Burkina Faso, 01 BP 1091 Bobo-Dioulasso \\ 01,E-mail:zouwiss@yahoo.fr, Cel. (+) 22670738033 \\ ${ }^{2}$ Centre National de la Recherche Scientifique et Technique, Institut de l'Environnement et de Recherches \\ Agricoles, Département Production Forestière, BP 10 Koudougou, Burkina Faso. \\ ${ }^{3}$ Directrice du Laboratoire d'Etudes et de Recherches des Ressources Naturelles et des Sciences de \\ l'Environnement (LERNSE), Université Polytechnique de Bobo-Dioulasso (UPB), Burkina Faso. 01 BP 1091 \\ Bobo-Dioulasso 01, E-mail : cykabore@yahoo.fr, Cel. (+) 22670723271 \\ ${ }^{*}$ Auteur correspondant, E-mail : zouwiss@yahoo.fr
}

\section{RESUME}

Dans la zone nord soudanienne du Burkina Faso, les gousses de Piliostigma reticulatum sont intensément utilisées pendant la saison sèche comme aliment d'appoint pour le bétail. L'objectif de cette étude est d'améliorer la qualité nutritionnelle de ces gousses pour optimiser leur apport. Neuf rations comprenant chacune des gousses de $P$. reticulatum (70 p.100) et du foin de Pennisetum pedicellatum (30 p.100) distribuées à raison de $50 \mathrm{~g} \mathrm{MS} / \mathrm{kg} \mathrm{p}^{0,75}$ ont été testées sur des ovins de race Djallonké. Les gousses ont subi différents traitements physiques (concassage, mouture) suivis ou non de traitement à l'urée auxquelles est associé dans certains cas du charbon de bambou à des doses de 0,25 ou $0,50 \mathrm{~g} / \mathrm{kg}$ PV dans le but d'influencer l'action des tanins. L'utilisation de l'urée a entraîné, quelle que soit la forme de distribution, une amélioration de la digestibilité, surtout celle des matières azotées en raison de l'augmentation significative de leur teneur. L'adjonction du charbon de bambou n'a amélioré la digestibilité des gousses non traitées à l'urée qu'à la dose de $0,50 \mathrm{~g} / \mathrm{kg} \mathrm{PV}$. Par contre, sur des gousses traitées à l'urée, il a permis une amélioration significative ( $<<0,05)$ de la digestibilité à la dose de $0,25 \mathrm{~g} / \mathrm{kg}$ PV.

(C) 2010 International Formulae Group. All rights reserved.

Mots clés : Concassage, broyage, urée, charbon de bambou, ovins.

\section{INTRODUCTION}

Piliostigma reticulatum (D.C.) Hochst est un ligneux sahélien et sahélo-soudanien. L'espèce est classée parmi les ligneux préférés des animaux dans le Sahel sénégalais (Cissé et al., 2002). Ces gousses sont connues pour leur bonne palatabilité (Issa et al., 2000). Dans les zones où les ressources fourragères sont déficientes, elles constituent une alternative dans l'alimentation des ruminants. C'est le cas dans la zone nord soudanienne du Burkina Faso, notamment dans les élevages péri-urbains. Les gousses de $P$. reticulatum font d'ailleurs dans cette localité, l'objet de commerce. Elles sont utilisées en nature dans des rations en association avec d'autres ingrédients. Les gousses de $P$. reticulatum comme fourrage ont l'avantage d'être 
disponibles et bon marché. L'activité de récolte occupe les femmes pendant une partie de la période post-culturale et leur procure ainsi un revenu permettant de subvenir aux besoins familiaux de première nécessité (Ouédraogo, 2006). Cependant, des études effectuées sur la valeur nutritive des fruits de $P$. reticulatum révèlent qu'elles sont peu digestibles surtout pour ce qui est de leurs matières azotées totales (Sanou, 2005). Cette faible digestibilité pourrait être liée soit à :

- la forme habituelle de présentation des gousses (concassage grossier), où les graines restent en majorité entières. En effet, ces graines résistent au processus de la digestion et sont rejetées intactes dans les fèces. Une étude réalisée sur une espèce voisine $(P$. thonningii) montre que les graines représentent 54 p.100 des teneurs en matières azotées totales des gousses entières (Ouédraogo, 2006) ;

- l'inaccessibilité des parois par les microorganismes due à leur structure très lignifiée et à leur degré de lignification (ADL/NDF) élevé et égal à 0,67 (Sanou, 2005). Cette teneur élevée en lignine (ADL), pourrait entraîner un blocage des matières azotées totales au niveau de la fraction lignocellulosique (Koné, 1987) conduisant à une réduction de la disponibilité en azote. De plus, une corrélation négative a été établie entre la digestibilité des fourrages et le taux de lignine (Casler et Jung, 2006);

- la présence de tanins qui sont en général présents dans les fourrages ligneux et qui les empêchent d'exprimer leur potentialité réelle (Andrabi et al., 2005 ; Mc Sweeney et al., 2005) en réduisant la protéolyse et la disponibilité de l'azote fermentescible pour l'activité du rumen (Min et al., 2005); condition nécessaire pour atteindre la digestibilité potentielle d'une ration et des niveaux d'ingestion élevés. Il existe d'ailleurs une forte corrélation négative d'une part, entre la digestibilité de la matière sèche et la teneur en tanins et d'autre part, entre la digestibilité des matières azotées et la teneur en tanins (Kaboré-Zoungrana, 1995).
De ce fait, l'amélioration de la valeur nutritive des gousses de $P$. reticulatum pour permettre une utilisation plus rationnelle de cette ressource fourragère et ainsi encourager la préservation de l'espèce, semble pertinente.

C'est pourquoi cette étude se propose d'examiner différentes méthodes d'amélioration de la digestibilité des gousses de $P$. reticulatum. Il s'agit:

- du traitement physique (concassage, broyage) des gousses;

- du traitement à l'urée qui est une alternative pour améliorer les teneurs en matières azotées des fourrages pauvres et;

- de l'adjonction du charbon de bambou (Oxythenanthera abyssinica) qui absorberait certains éléments végétaux secondaires et améliorerait la valeur nutritive du fourrage.

\section{MATERIEL ET METHODES}

Concassage, broyage et traitement des gousses à l'urée

Les gousses sèches de $P$. reticulatum ont subit deux types de traitement physique : le concassage dans un mortier par des femmes suivi ou non d'un broyage dans un moulin à la grille de $8 \mathrm{~mm}$. Les gousses concassées ou moulues ont été traitées par la suite à l'urée.

Pour le traitement, on a utilisé l'urée comme générateur de l'ammoniac qui est une source d'azote fermentescible, nécessaire au bon fonctionnement des microorganismes (Ndlovu, 1992). Le traitement a été effectué à la dose de 4 p.100. L'urée a été apportée à 4 $\mathrm{kg}$. Cette quantité a été préalablement dissoute dans de l'eau à raison de $4 \mathrm{~kg}$ pour 501 d'eau. Le traitement a concerné à la fois des quantités de $15 \mathrm{~kg}$ de gousses concassées ou broyées qui sont par la suite tassées, fermées et ensuite déposées dans un sac plastique qui est à son tour fermé et stocké pendant quatre (4) semaines.

\section{Adjonction du charbon de bambou}

Du charbon de bambou a été d'abord écrasé à la grille de $2 \mathrm{~mm}$, puis mélangé aux gousses traitées ou non à l'urée au moment de leur distribution aux animaux. Deux doses : 0,25 et $0,5 \mathrm{~g} / \mathrm{kg} \mathrm{PV}$ de l'animal ont été 
utilisées afin d'apprécier leur effet sur l'utilisation digestive des différents composants des gousses traitées ou non à l'urée.

\section{Digestibilité in vivo}

Les expériences ont porté sur des ovins de race Djallonké, variété locale Mossi. Les animaux sont des mâles adultes castrés de poids vif variant entre $15 \mathrm{~kg}$ et $27 \mathrm{~kg}$. Ils ont été repartis en lots homogènes de six (6) animaux selon le poids vif. Tous les animaux sont maintenus dans des cages individuelles à métabolisme. L'essai de la digestibilité a duré 21 jours, dont 14 jours d'adaptation des animaux aux régimes et sept (7) jours de collecte intégrale des fecès. Les quantités ingérées et refusées de même que les fecès ont été pesées et échantillonnées quotidiennement pour des analyses au laboratoire.

Chaque lot a été alimenté en quantité limitée à raison de $50 \mathrm{~g} \mathrm{MS} / \mathrm{kg}$ de poids métabolique $\left(\mathrm{kg} \mathrm{P}^{0,75}\right)$. La ration a été composée de gousses de $P$. reticulatum à 70 p.100 et de foin de Pennisetum pedicellatum à 30 p. 100 sur la base de la MS. Les aliments ont été distribués deux fois par jour, le matin et le soir, et les animaux ont disposé d'eau et de pierre à lécher à volonté. Neuf (9) régimes ont fait l'objet de cette mesure de l'utilisation digestive :

- gousses concassées+ foin ;

- gousses concassées + foin +charbon $0,25 \mathrm{~g} / \mathrm{kg} \mathrm{PV}$;

- gousses concassées +foin+ charbon $0,50 \mathrm{~g} / \mathrm{kg} \mathrm{PV}$;

- gousses concassées traitées à l'urée + foin ;

- gousses broyées+ foin ;

- gousses broyées + foin +charbon $0,25 \mathrm{~g} / \mathrm{kg} \mathrm{PV}$;

- gousses broyées traitées à l'urée+ foin ;

- gousses broyées traitées à l'urée + foin + charbon $0,25 \mathrm{~g} / \mathrm{kg} \mathrm{PV}$;

- gousses broyées traitées à l'urée + foin +charbon $0,5 \mathrm{~g} / \mathrm{kg} \mathrm{PV}$.

\section{Analyses bromatologiques}

Des échantillons de gousses et fecès prélevés chaque jour, ont été séchés à l'étuve à $55^{\circ} \mathrm{C}$ pendant 24 heures et broyés à la grille de $1 \mathrm{~mm}$ pour constituer des échantillons moyens pour la période de collecte. Des analyses chimiques classiques ont été effectuées : matière sèche (MS) à l'étuve à $105{ }^{\circ} \mathrm{C}$ pendant $24 \mathrm{~h}$; cendres totales (MM) par calcination de la matière sèche à $550{ }^{\circ} \mathrm{C}$; matières azotées totales (MAT) correspondant à l'azoté selon Kjeldahl $(\mathrm{N} \times 6,25)$; constituants pariétaux, NDF (neutal detergent fiber), $\mathrm{ADF}$ (acid detergent fiber) dosé directement sur l'échantillon et ADL (acid detergent lignin) sulfurique déterminé à partir de l'ADF.

Méthodes de calcul de la digestibilité

Les coefficients d'utilisation digestive des divers constituants (MS, MO, MAT, NDF, $\mathrm{ADF})$ pour les différents régimes étudiés sont déterminés.

\section{Analyse des données}

Les données de digestibilité ont été soumises à une analyse de variance au moyen du logiciel STATGRAPHICS. Les différences de moyenne de CUD suivant les traitements ont été considérées comme significatives au seuil de $5 \%$.

\section{RESULTATS}

\section{Composition chimique des aliments}

Les gousses ont une teneur relativement faible en MAT et des teneurs en constituants pariétaux élevés surtout pour ce qui concerne la lignine (Tableau 1). Après le traitement à l'urée, les MAT ont augmenté de $155 \%$ pour des gousses concassées et $171 \%$ pour celles broyées. Le traitement à l'urée a modifié les teneurs en constituants pariétaux des gousses. L'augmentation la plus importante est notée au niveau des teneurs en NDF respectivement de 5 et 3 p. 100 pour les gousses concassées et moulues.

Digestibilité des gousses et effet des différents traitements Effet du traitement physique

La digestibilité des gousses sans distinction de la forme de distribution est faible pour tous les paramètres mesurés (MS, MO, MA, NDF, ADF). Néanmoins, les différents coefficients d'utilisation digestive pour tous les constituants des gousses concassées sont plus élevés que ceux des 
gousses broyées (Tableau 2); les différences ne sont cependant pas significatives au seuil de 5\%. Le broyage a donc entraîné une baisse de la digestibilité pour tous les paramètres considérés. Cette dépréciation est surtout plus marquée pour les matières azotées.

\section{Effet du traitement à l'urée}

Avec les gousses concassées traitées à l'urée, une augmentation significative ( $\mathrm{p}<$ $0,05)$ de l'utilisation digestive a été notée uniquement pour la MO (dMO) et les MA (dMA). La dMO a été améliorée de $101 \mathrm{~g} / \mathrm{kg}$ MS et la dMA de $228 \mathrm{~g} / \mathrm{kg}$ MS (Tableau 3). Une amélioration significative $(\mathrm{p}<0,05)$ de la digestibilité des gousses broyées a été notée. Les valeurs ont augmenté de $32 \mathrm{~g} / \mathrm{kg}$ MS pour la dMS, $62 \mathrm{~g} / \mathrm{kg}$ MS pour la dMO et $306 \mathrm{~g} / \mathrm{kg}$ MS pour la dMA. En revanche, une diminution significative $(\mathrm{p}<0,05)$ de la digestibilité des parois totales a été observée.

\section{Effet du charbon de bambou}

L'adjonction de $0,25 \mathrm{~g} / \mathrm{kg} \quad \mathrm{PV} \quad \mathrm{du}$ charbon de bambou aux gousses non traitées à l'urée, n'a pas permis une amélioration de la digestibilité de la ration. En revanche, une amélioration significative de la digestibilité a été observée à la dose de $0,50 \mathrm{~g} / \mathrm{kg}$ PV avec les gousses concassées (Tableau 4). Si l'effet du charbon à la dose $0,25 \mathrm{~g} / \mathrm{kg}$ PV n'a eu aucun effet positif sur la digestibilité des gousses de $P$. reticulatum, une synergie d'action est par contre enregistrée entre urée et charbon lorsque les gousses sont préalablement traitées. L'augmentation des teneurs digestibles a été de $22,114,430$ et 41 $\mathrm{g} / \mathrm{kg}$ MS respectivement pour la MS, la MO, les MAT et les NDF (Tableau 5). L'effet favorable de l'urée sur l'utilisation du charbon lorsque ce dernier est augmenté à raison de $0,50 \mathrm{~g} / \mathrm{kg} \mathrm{PV}$, n'est retrouvé que pour les parois totales.

Tableau 1 : Composition chimique $(\mathrm{g} / \mathrm{kg} \mathrm{MS})$ des gousses de $P$. reticulatum avant et après traitement à l'urée.

\begin{tabular}{lcccccccc}
\hline & MS & MO & MAT & NDF & ADF & ADL & Hemicelluloses & Cellulose \\
\hline GC Non traitées & 890,8 & 945,7 & 76,7 & 537,6 & 390,9 & 256,9 & 146,7 & 134 \\
GC traitées & 594,9 & 942 & 193,9 & 586,3 & 422,1 & 281,4 & 164,2 & 140,7 \\
GB Non traitées & 898 & 944,6 & 79,2 & 542,8 & 399,4 & 265,8 & 143,4 & 133,6 \\
GB traitées & 587,2 & 804,3 & 214,8 & 572,8 & 422,5 & 281,7 & 150,3 & 140,8 \\
\hline
\end{tabular}

GC: gousses concassées; GB:gousses broyées.

Tableau 2: Effet du traitement physique sur la digestibilité de la ration (g/kg MS).

\begin{tabular}{lccccc}
\hline Aliments & dMS & dMO & dMA & dNDF & dADF \\
\hline GC+ foin & $500(8,2)^{\mathrm{a}}$ & $512(9,1)^{\mathrm{a}}$ & $352(19)^{\mathrm{a}}$ & $417(15,2)^{\mathrm{a}}$ & $145(26)^{\mathrm{a}}$ \\
$\mathrm{GB}+$ foin & $469(8,2)^{\mathrm{a}}$ & $477(8,5)^{\mathrm{a}}$ & $281(12)^{\mathrm{a}}$ & $371(21)^{\mathrm{a}}$ & $138(12)^{\mathrm{a}}$ \\
\hline
\end{tabular}

Les valeurs entre parenthèse indiquent les erreurs standard; GC: gousses concassées; GB:gousses broyées. Les moyennes de digestibilité de la ration de la même colonne affectées des mêmes lettres ne diffèrent pas significativement $(\mathrm{p}<0,05)$.

Tableau 3: Effet du traitement à l'urée sur la quantité de matières digestibles ( $\mathrm{g} / \mathrm{kg}$ MS) des différentes rations contenant les gousses.

\begin{tabular}{lccccc}
\hline Aliments & dMS & dMO & dMA & dNDF & dADF \\
\hline Ration:foin+gousses & & & & & \\
GC sans urée & $500(8,2)^{\mathrm{b}}$ & $512(9,1)^{\mathrm{b}}$ & $352(19)^{\mathrm{b}}$ & $417(15,2)^{\mathrm{b}}$ & $145(26)^{\mathrm{a}}$ \\
GC avec urée & $453(10,3)^{\mathrm{a}}$ & $613(3,9)^{\mathrm{c}}$ & $580(6,9)^{\mathrm{c}}$ & $357(2,6)^{\mathrm{ab}}$ & $150(19,3)^{\mathrm{a}}$ \\
GB sans urée & $469(8,1)^{\mathrm{ab}}$ & $477(8,5)^{\mathrm{a}}$ & $281(12)^{\mathrm{a}}$ & $371(21)^{\mathrm{ab}}$ & $138(11,7)^{\mathrm{a}}$ \\
GB avec urée & $501(4,5)^{\mathrm{b}}$ & $539(4)^{\mathrm{b}}$ & $587(3,6)^{\mathrm{c}}$ & $309(13,7)^{\mathrm{a}}$ & $106(32)^{\mathrm{a}}$ \\
\hline \multicolumn{2}{l}{ Les valeurs entre parenthèse indiquent les erreurs standard ; GC: gousses concassées; GB:gousses broyées. Les moyennes }
\end{tabular}
de digestibilité de la même colonne affectées des mêmes lettres ne diffèrent pas significativement $(\mathrm{p}<0,05)$. 
Tableau 4 : Effet de l'adjonction du charbon de bambou sur la digestibilité (g/kg MS) des gousses concassées (a) et broyées (b).

(a)

\begin{tabular}{lccccc}
\hline Aliments & dMS & dMO & dMA & dNDF & dADF \\
\hline Ration:foin+gousses & & & & & \\
GC sans ch. & $500(8,2)^{\mathrm{a}}$ & $512(9,1)^{\mathrm{a}}$ & $352(19)^{\mathrm{ab}}$ & $417(15,2)^{\mathrm{b}}$ & $145(26)^{\mathrm{ab}}$ \\
GC ch. 0,25 & $488(7,2)^{\mathrm{a}}$ & $500(6,3)^{\mathrm{a}}$ & $305(17)^{\mathrm{a}}$ & $347(19)^{\mathrm{a}}$ & $77(17)^{\mathrm{a}}$ \\
GC ch. 0,50 & $545(3,4)^{\mathrm{b}}$ & $547(3,3)^{\mathrm{b}}$ & $403(14)^{\mathrm{b}}$ & $470(4,5)^{\mathrm{b}}$ & $170(15)^{\mathrm{b}}$ \\
\hline (b) & & & & & \\
\hline GB sans ch. & $469(8,2)^{\mathrm{a}}$ & $477(8,5)^{\mathrm{a}}$ & $281(12)^{\mathrm{a}}$ & $371(21)^{\mathrm{a}}$ & $138(12)^{\mathrm{a}}$ \\
GB ch. 0,25 & $468(5,1)^{\mathrm{a}}$ & $478(5,7)^{\mathrm{a}}$ & $240(14)^{\mathrm{a}}$ & $349(9,4)^{\mathrm{a}}$ & $82(4,1)^{\mathrm{b}}$ \\
\hline
\end{tabular}

Les valeurs entre parenthèse indiquent les erreurs standard ; ch. Charbon ; $0,25: 0,25 \mathrm{~g} / \mathrm{kg} \mathrm{PV} ; 0,50: 0,50 \mathrm{~g} / \mathrm{kg} \mathrm{PV} ; \mathrm{GC}$ : gousses concassées ; GB : gousses broyées. Les moyennes de digestibilité de la même colonne affectées des mêmes lettres ne diffèrent pas significativement $(\mathrm{p}<0,05)$.

Tableau 5 : Effet du traitement à l'urée sur l'adjonction du charbon de bambou dans les rations contenant des gousses (g/kg MS).

\begin{tabular}{lccccc}
\hline Aliments & dMS & dMO & dMA & dNDF & dADF \\
\hline Ration:foin+gousses & & & & & \\
GB urée & $501(4,5)^{\mathrm{b}}$ & $539(4)^{\mathrm{ab}}$ & $587(3,6)^{\mathrm{b}}$ & $309(14)^{\mathrm{a}}$ & $106(32)^{\mathrm{ab}}$ \\
GB ch. 0,25 & $468(5,1)^{\mathrm{ab}}$ & $478(5,7)^{\mathrm{a}}$ & $240(14)^{\mathrm{a}}$ & $349(9,4)^{\mathrm{ab}}$ & $82(4,1)^{\mathrm{ab}}$ \\
GB urée + ch. 0,25 & $490(6,7)^{\mathrm{ab}}$ & $592(38)^{\mathrm{b}}$ & $670(11)^{\mathrm{c}}$ & $382(8,7)^{\mathrm{c}}$ & $170(27)^{\mathrm{b}}$ \\
GB urée + ch. 0,50 & $449(20)^{\mathrm{a}}$ & $547(26)^{\mathrm{ab}}$ & $594(16)^{\mathrm{b}}$ & $369(9,8)^{\mathrm{c}}$ & $50(20)^{\mathrm{a}}$ \\
\hline \multicolumn{2}{l}{ Les valeurs entre parenthèse indiquent les erreurs standard ; ch. : Charbon $; 0,25: 0,25 \mathrm{~g} / \mathrm{kg}$ PV; $0,5: 0,5 \mathrm{~g} / \mathrm{kg} \mathrm{PV} ;$} \\
GB: gousses broyées. Les moyennes de digestibilité de la même colonne affectées des mêmes lettres ne diffèrent \\
pas significativement $(\mathrm{p}<0,05)$.
\end{tabular}

\section{DISCUSSION}

Effet de l'urée sur la composition chimique

Le traitement à l'uré a permis de doubler les teneurs en MAT. Ces augmentations ont été conformes à celles rapportées par d'autres auteurs (MichaletDoreau et Guedes, 1989; Nyarko-Badohu et al., 1993; Tengyun, 2000; Hassoun et Latchimy, 2002). Cet accroissement de la teneur en MAT est due à la fixation de l'azote issu de l'uréolyse sur le fourrage; fixation pouvant atteindre $450 \mathrm{~g} / \mathrm{kg}$ de l'azote total libéré par la réaction d'uréolyse (MichaletDoreau et Guedes, 1989).

Les alcalis dont l'urée, altèrent la structure physique des parois cellulaires des fourrages traités, modifiant ainsi la structure biochimique de ces parois (Cordesse, 1987). Les teneurs élevées en NDF et ADF obtenues après traitement à l'urée sont aussi notées par
Vitti et al. (2005). L'augmentation de la teneur en NDF a été en contradiction avec ceux de Chenost et Dulphy (1987), NyarkoBadohu et al. (1993) et Tengyun (2000), probablement liée aux conditions de traitement. Pour la fraction ADF, l'augmentation de sa teneur est classiquement observée (Michalet-Doreau et Guedes, 1989; Tengyun, 2000 ; Hassoun et Latchimy, 2002).

L'effet du traitement à l'urée sur les composants des parois cellulaires serait lié à l'humidité du traitement. Dans des conditions d'humidité inférieure, les teneurs en parois diminuent du fait d'une augmentation de la fixation de l'azote sur ces parois (MichaletDoreau et Guedes, 1989). En revanche, lorsque l'humidité est élevée, les teneurs en parois totales augmentent. Cette augmentation est liée à la formation de produits (réaction de Maillard) non solubilisés, lors du dosage aux 
détergents neutres (Scheineider et Flachowsly, 1990). Une autre raison serait que des éléments solubles en présence d'une quantité importante d'eau soient solubilisés (Ben Salem et al., 2005a) et entraînés vers le fond lors du traitement (Larwence et al., 2000). Ce qui expliquerait l'augmentation plus importante des parois des gousses concassées. Le dépôt sous forme d'une pâte observé sur les parois et fond des sacs de traitement des gousses concassées serait constitué de ces éléments solubilisés et entraînés. Ce phénomène n'est pas observé avec les gousses broyées probablement parce qu'elles sont plus homogènes.

L'augmentation de la cellulose a été inférieure à celle mentionnée par (Hassoun et Latchimy, 2002) et l'accroissement de la teneur en hémicelluloses est en contradiction avec les résultats de certains travaux (Michalet-Doreau et Guedes, 1989; NyarkoBadohu et al., 1993; Hassoun et Latchimy, 2002 ; Mesfin et Ledin, 2004). Ces résultats indiquent que dans notre étude, la brisure de la liaison lignocellulose lors du traitement à l'urée n'a pas été suffisante pour libérer une bonne partie de la cellulose (Wambui et al., 2006).

\section{Effet du traitement physique sur la digestibilité}

La digestibilité des gousses distribuées sans aucun traitement est relativement faible. Cette faible digestibilité peut être assignée au faible apport en azote du à l'inaccessibilité des graines au processus de digestion, à la forte lignification des gousses et à la présence éventuelle de tanins. Le broyage des gousses qui rend certes disponible l'azote contenu dans les graines, déprécie la digestibilité. Ce résultat est conforme à ceux observés par (Rezaeian et al., 2006). La baisse de la digestibilité serait liée (i) à la structure peutêtre trop fine des gousses $(8 \mathrm{~mm})$, qui réduirait le temps de séjour des particules dans le rumen (Scheineider et Flachowsly, 1990); (ii) à la libération de certains facteurs antinutritionnels (tanins) qui sont malheureusement en teneur souvent élevée dans les gousses de certains ligneux (Mlambo et al., 2008).

\section{Effet du traitement à l'urée sur la digestibilité}

Le traitement des gousses broyées a entraîné une augmentation significative de la dMS, de la dMO et de la dMA comme cela a pu être noté par ailleurs sur divers types de fourrages (Hassoun et Latchimy, 2002).

La digestibilité des MA (dMA) a significativement augmenté grâce aux concentrations en ammoniac du rumen, consécutive au traitement qui permettent une meilleure activité de la microflore (Kusmartono, 2002). Cela se traduit par l'amélioration des conditions du rumen où les nutriments, surtout l'azote soluble sont disponibles en permanence (Ndlovu, 1992, Fric, 2004). Cette amélioration de la digestibilité pourrait être aussi imputable à la réduction des teneurs en tanins voire leur inhibition dans certains cas, suite au traitement à l'urée (Ben Salem et al., 2005b et Vitti et al., 2005). Cette vertu de l'urée serait liée au caractère alcalin qu'il confère à l'aliment lors du traitement et à la formation de bicarbonate (Van-Soest, 2006). L'effet des alcalis sur l'hydrolyse des tanins a été mis en évidence sur les feuilles de Acacia cyanophyla trempées dans une solution (cendre de bois de la même espèce + eau) de pH 12 (Ben Salem et al., 2005a). Cette opération a entraîné une réduction significative des teneurs en tanins totaux et en tanins condensés. Une réduction de concentration en tanins de $920 \mathrm{~g} / \mathrm{kg}$ MS après traitement aux alcalis est par ailleurs notée (Alam et al., 2005). Il a aussi été établi qu'à un $\mathrm{pH}$ alcalin voisin de 8 (Rakhamani et al., 2005) ou supérieur à 7,5 de même qu'à des $\mathrm{pH}$ acide inférieurs à 3,5 (Ben Salem et al., 2005b), on assisterait à une solubilisation des complexes protéines-tanins.

Le traitement à l'urée n'a pas permis une amélioration de la digestibilité des parois totales comme préconisée par Wambui et al. (2006). La dépréciation observée de la 
digestibilité des parois est de même ordre que celle observée par Chivandi et al. (2007). Elle serait due aux teneurs élevées en NDF (Ndlovu, 1992), en lignocellulose et/ou à la perte par volatilisation des molécules susceptibles de casser les liaisons lignocellulosiques lors du traitement à l'urée et en augmenter ainsi la digestibilité des parois (Wambui et al., 2006).

\section{Effet du charbon de bambou}

L'adjonction du charbon de bambou a entraîné une diminution de la digestibilité sur des gousses non traitées à l'urée. Cette dépréciation a été moins importante pour les gousses broyées. Le broyage casserait probablement des liaisons faisant intervenir les tanins, pour les libérer et favoriser l'action $\mathrm{du}$ charbon sur ces derniers. L'adjonction du charbon de Bambou à la dose de $0,25 \mathrm{~g} / \mathrm{kg}$ PV quelle que soit la forme de distribution, n'a pas amélioré la digestibilité des gousses. En revanche, la même dose a entrâné une augmentation de la digestibilité des feuilles de Khaya senegalensis (Ouédraogo, 2006). Cela pourrait témoigner de la nature des composés secondaires et de leurs teneurs différentes d'une espèce à l'autre. Sur les gousses concassées, la dose $0,50 \mathrm{~g} / \mathrm{kg}$ PV a amélioré significativement la digestibilité. Il a été également montré que la dose $0,50 \mathrm{~g} / \mathrm{kg} \mathrm{PV}$ a une incidence positive sur la digestibilité des feuilles de Acacia mangium (Van et al., 2006). La réponse enregistrée lors de l'adjonction du charbon serait non seulement fonction de la dose administrée mais aussi du type de tanin et du rapport tanin/charbon.

L'adjonction du charbon à la dose de $0,25 \mathrm{~g} / \mathrm{kg} \mathrm{PV}$ aux gousses traitées à l'urée, a amélioré la dMS, dMO, dMA et de la digestibilité des parois (dNDF et dADF). Le traitement à l'urée favorise l'action du charbon sur les tanins. En effet, l'alcalinité du traitement à l'urée libère les groupements phénoliques des tanins (Morrison, 1974 cité par Cordesse, 1987) et facilite leur absorption par le charbon. L'amélioration de la digestibilité des MA suite à l'ajout du charbon a été la plus importante. Cette meilleure valorisation de l'azote s'expliquerait par le fait que le charbon a la propriété de former avec les composés phénoliques des complexes et empêchent ainsi l'interférence des tanins sur les enzymes protéolytiques. De ce fait, le charbon de bambou augmenterait la disponibilité de certains macro-nutriments, particulièrement les protéines, les rendant accessibles au processus de la digestion (Van et al., 2006).

Le doublement de la dose de charbon (de 0,25 à $0,50 \mathrm{~g} / \mathrm{kg} \mathrm{PV}$ ) associé au traitement à l'urée par rapport à la dose $0,25 \mathrm{~g} / \mathrm{kg}$ PV a conduit à des digestibilités plus faibles indiquant ainsi qu'au delà d'un certain niveau de charbon, la digestion (Kutlu et al., 2001) voire parfois le métabolisme ruminal (Van et al., 2006) peuvent être perturbés.

\section{Conclusion}

Le traitement à l'urée et l'adjonction du charbon de bambou constitue une alternative pratique, économique et efficace pour rendre plus digestes les gousses de $P$. reticulatum. Tout en améliorant les teneurs en azote et probablement en inhibant les tanins, ils permettent une meilleure valorisation des gousses. Ces technologies sont simples à mettre en œuvre car elles nécessitent un matériel limité, facile à se procurer et d'un coût modeste. L'adjonction du charbon de bambou aux doses de $0,50 \mathrm{~g} / \mathrm{kg}$ PV sur les gousses de $P$. reticulatum et de $0,25 \mathrm{~g} / \mathrm{kg}$ PV sur celles traitées à l'urée peuvent être recommandées car trouvées efficaces pour améliorer la digestibilité. Compte tenu de l'importance de l'alimentation en production animale, la valorisation de ces gousses, localement disponibles et bon marché, pourrait réduire le coût de production et par conséquent améliorer la marge bénéficiaire du producteur. Il restera à s'assurer de l'effet bénéfique à long terme de l'utilisation du charbon en production animale (croissance et engraissement). Aussi, cette valorisation des gousses devrait entraîner une utilisation de quantités plus faibles en production animale et 
réduire de ce fait la pression qui est exercée sur cette ressource au niveau du pâturage.

\section{REFERENCES}

Alam MR, Kabir AKMA, Amin MR, Mcneill DM. 2005. The effect of calcium hydroxide treatment on the nutritive and feedind value of Albizia procera for growing goats. Animal Feed Science and Technology, 122: 135-148.

Andrabi SM, Ritchie MM, Strinson C, Horazdagoda A, Hyde M, McNeill DM. 2005. In vivo assessment of the ability of condensed tannins to interfere with the digestibility of plant protein in sheep. Animal Feed Science and Technology, 122: 13-27.

Ben Salem H, Abidi S, Makkar HPS, Nefzaoui A. 2005a. Wood ash treatment a cost-effective way to desactivate tannins in Acacia cyanophyla Lindl. Foliage and to improve digestion by Barbarine sheep. Animal Feed Science and Technology, 122: 93-108.

Ben Salem H, Saghrouni L, Nefzaoui A. 2005b. Attemps to desactivate tannins in fodder shrubs with physical and chemical treatments. Animal Feed Science and Technology, 122: 109-121.

Casler MD, Jung HJG. 2006. Relationships of fibre, lignin and phenolics to in vitro fibre digestibility in three perennical grasses. Animal Feed Science and Technology, 125: 151-161.

Chenost M, Dulphy JP. 1987. Amélioration de la valeur alimentaire (composition, digestibilité, ingestibilité) des mauvais foins et des pailles par les différents types de traitement. In Les Fourrages Secs: Récolte, Traitement, Utilisation. Ed. INRA: Paris; 199-230.

Chivandi E, Chipfupa L, Ncube S. 2007. In situ dry matter and nitrogen degradability of ammonium nitrate and urea treated dry veld grass and Zea mays stover in mature goats. Livestock Research for Rural Development, 19(2). http://www.lrrd.org/ lrrd19/2/chiv19028.htm
Cisse M, Ly I, Nianogo AJ, Sane I, Sawadogo JG. 2002. Grazing behaviour and milk yield of senegalese Sahel goat. Small Ruminant Research, 43: 85-95.

Cordesse R. 1987. Technologie du traitement des pailles à l'ammoniac. In Les Fourrages Secs: Récolte, Traitement, Utilisation. Ed. INRA : Paris ; 131-142.

Fric D. 2004. Dynamique de digestion et point de vue de l'animal. Actes de séminaire sur les recherches en Agriculture Biologiques. Actes de la 3 ème journée technique du pôle scientifique en Agriculture Biologique du Massif Central, Tulles-Naves, le 6 Novembre 2003 sur «les fourrages en Agriculture Biologique dans le Massif Central». Ed. INRA-ACTA. Site web: www.itab.asso. fr./poleABMassifCentral.htm.

Hassoun P, Latchimy JY. 2002. Traitement du foin de Chloris gayana à l'ammoniac généré à partir de l'urée. Résultats d'un essai en ferme à l'île de la Réunion. Livestock Research for Rural Development, 14(4). http://www.lrrd. org/lrrd14/4/hass144.htm

Issa S, Yakouba, Djibrillou OA, Bachir O, Abdou N, Karao M. 2000. Foraging behaviour of cattle, sheep and goats in Sidi Koira, Niger River Valley. In Production and Utilisation of MultiPupose Fodder Shrubs and Trees in West Asia, North Africa and the Sahel. ICARDA, Aleppo, Syria, ILRI: Narobi, Kenya, Viii + p. 60 .

Kabore-Zoungrana CY. 1995. Composition chimique et valeur nutritive des herbacées et ligneux des pâturages naturels soudaniens et des sous produits du Burkina Faso. Thèse de Doctorat d'Etat, Université de Ouagadougou, p. 201.

Koné AR. 1987. Valeur alimentaire des ligneux fourragers des régions sahélienne et soudanienne d'Afrique occidentale: Recherche d'une méthode simple d'estimation de la digestibilité et de la valeur azotée. Thèse de $3 \mathrm{e}$ cycle, Université de Paris VI-IEMVT, p. 205. 
Kusmartono 2002. Effect of supplementing Jackfruit (Artocarpus heterophyllus L.) wastes with urea or leaves of Gliricidia sepium on feed intake and digestibility in sheep and steers. Livestock Research for Rural Development, 14(2). http://www. lrrd.org/lrrd14/2/kusm142.htm

Kutlu HR, Ünsal I, Gôrgülü M. 2001. Effects of providing dietary wood (oak) charcoal to broiler chicks and laying hens. Animal Feed Science and Technology, 90: 213226.

Larwence A, Saddeck T, Chabaca R. 2000. Proposition d'une méthode subhumide de traitement des pailles à l'urée. INRA. EDP Sciences. Annale de Zootechnie, 49: 479-485.

McSweeney CS, Gough J, Conlan LL, Hegarty MP, Palmer B, Krause DO. 2005. Nutritive value assessment of the tropical shrubs legume Acacia angustissima: anti-nutritional compounds and in vitro digestibility. Animal Feed Science and Technology, 121: 175-190.

Mesfin R, Ledin I. 2004. Comparison of feeding urea-treated teff and barley straw based diets with hay based diet to cross bred dairy cows on feed intake, milk yield, milk composition and economic benefits. Livestock Research for Rural Development, 16(12). www.lrrd.org/ lrrd16/12/mesf16104.htm

Michalet-Doreau B, Guedes CVM. 1989. Nutritive value assessment of the tropical shrubs legume Acacia angustissima: antinutritional compounds and in vitro digestibility. Annale de Zootechnie, 38: 259-288.

Min BR, Attwood GT, McNabb WC, Molan AL, Barry TN. 2005. The effect of condensed tannins from Lotus corniculatus on the proteolytic activities and growth of rumen bacteria. Animal Feed Science and Technology, 121(1-2): 45-58.

Mlambo V, Mould FL, Sikosana JLN, Smith T, Owen E, Mueller-Harvey I. 2008. Chemical composition and in vitro fermentation of tannin-rich tree fruits. Animal Feed Science and Technology, 140: 402-417.

Ndlovu LR. 1992. Complementarity of forages in ruminant digestion: theoretical considerations. In The complementary of feed resources for animal production in Africa proceeding. Ed. African Feeds Research Network ILCA (International Livestock Centre for Africa): Addis Ababa, Ethiopia; 430.

Nyarko-Badohu DK, Kayouli C, BA AA, Gasmi A. 1993. Valorisation des pailles de céréales en alimentation des ovins dans le Nord de la Tunisie: traitement aux alcalis (ammoniac/urée)-complémentation par des blocs melasse-urée. Livestock Research for Rural Development, 5(1). http://www.cipav.org.co/lrrd/lrrd5/1/tunis .htm

Ouedraogo S. 2006. Potentialités fourragères et essais d'amélioration de la valeur nutritive de trois ligneux fourragers: Piliostigma thonningii Schumach MileRedh, Piliostigma reticulatum (D.C.) Hoscht et Khaya senegalensis (Desr.) A. Juss. Mémoire de fin d'études, Université Polytechnique de Bobo-Dioulasso/IDR Burkina Faso, p. 67.

Rakhmani S, Brooker JD, Jones GP, Palmer B. 2005. Composition of condensed tannins from Calliandra calothyrsus and correlation with in sacco digestibility. Animal Feed Science and Technology, 121: 251-264.

Rezaeian M, Beakes GW, Chaudhry AS. 2006. Effect of feeding chopped and pelleted Lucerne on rumen fungal fermentation profiles and in sacco degradation of barley straw in sheep Animal Feed Science and Technology, 128: 292-306.

Sanou S. 2005 Piliostigma reticulatum (D.C.) Hoscht: Potentialités fourragères et essai d'amélioration de la Valeur nutritive des gousses Mémoire de fin d'études, Université Polytechnique de BoboDioulasso/IDR, Burkina Faso, p. 57. 
Schneider M, Flachowsly G. 1990. Studies on ammoniac treatment of wheat straw: effects of level of ammonia, moisture content. Treatment time and temperature on straw composition and degradation in the rumen of sheep. Animal Feed Science and Technology, 29: 251-264.

Tengyun G. 2000. Treatment and utilization of crop, straw and stover in China. Livestock Research for Rural Development, 14(12). http://www.lrrd. org/lrrd12/1/gao121.htm

Van DTT, Mui NT, Ledin I. 2006. Effect of method of processing foliage of Acacia mangium and inclusion of bamboo charcoal in the diet on performance of growing goats. Animal Feed Science and Technology, 130: 242-256.
Van Soest JP. 2006. Rice straw, the role of silica and treatments to improve quality. Animal Feed Science and Technology, 130: 137-171.

Vitti DMSS Nozetla EF, Addala AL, Bueno ICS, Silva Filho JC, Costa C, Bueno MS, Longo C, Vieira MEQ, Cabral Filho SLS, Godoy PB, Mueller-Harvey I. 2005. The effect of drying and urea treatment on nutritional and anti-nutritional components of browses collected during wet and dry seasons. Animal Feed Science and Technology, 122: 123-133.

Wambui CC, Abdulrazac SA, Noordin Q. 2006. The effect of supplementing urea treated maize stover with Tithonia, Calliandra and Sesbania to growing goats. Livestock Research for Rural Development, 18(5). 